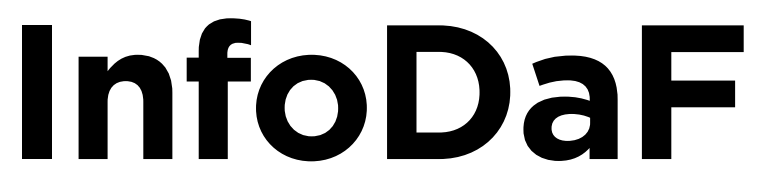

INFORMATIONEN DEUTSCH ALS FREMDSPRACHE

\title{
HERAUSGEGEBEN VOM
}

Deutschen Akademischen Austauschdienst

IN ZUSAMMENARBEIT MIT DEM

Fachverband Deutsch als Fremd- und Zweitsprache

VERANTWORTLICHER REDAKTEUR

Christian Krekeler, Konstanz

\section{REDAKTION}

Hebatallah Fathy (DAAD)

Lutz Köster, Bielefeld (verantwortlich für Rezensionen)

Uwe Koreik, Bielefeld

Christian Krekeler, Konstanz

Nicole Marx, Bremen

Fritz Neubauer, Bielefeld $\dagger$

Thorsten Roelcke, Berlin

Dietmar Rösler, Gießen

Michael Schart, Tokio

Barbara Schmenk, Waterloo, ON

\section{DE GRUYTER}


ABSTRACTED/INDEXED IN Service, Baidu Scholar, Celdes, CNKI Scholar (China National Knowledge Infrastructure), CNPIEC, BSCO Discovery Service, Google Scholar, J-Gate, KESLI-NDSL, (Korean National Discovery for Science Leaders), Naviga (Softweco), Primo Central (ExLibris), ReadCube, Summon (Serials Solutions/ProQuest), TDNet, WanFang Data, WorldCat (OCLC)

ISSN 0724-9616 · e-ISSN 2511-0853

Alle Informationen zur Zeitschrift, wie Hinweise für Autoren, Open Access, Bezugsbedingungen und Bestellformulare, sind online zu finden unter www.degruyter.com/view/j/infodaf

VERANTWORTLICHER REDAKTEUR Prof. Dr. Christian Krekeler, Hochschule Konstanz Technik, Wirtschaft und Gestaltung, Studienkolleg Konstanz, Brauneggerstr. 55, 78462 Konstanz, Tel.:

+49(0)7531 206-360, E-Mail: krekeler@htwg-konstanz.de

JOURNAL MANAGER Esther Markus, De Gruyter, Genthiner Straße 13, 10785 Berlin, Deutschland, Tel.: +49 (0)30 260 05-127, Fax: +49 (0)30 260 05-250,

E-Mail: esther.markus@degruyter.com

ANZEIGENVERANTWORTLICHE Claudia Neumann, De Gruyter, Genthiner Straße 13, 10785 Berlin, Deutschland, Tel.: +49 (0)30 260 05-226, Fax: +49 (0) 30260 05-264

E-Mail: anzeigen@degruyter.com

(C) 2019 Walter de Gruyter GmbH, Berlin/Boston

SATZ jürgen ullrich typosatz, Nördlingen

DRUCK Franz X. Stückle Druck und Verlag e.K., Ettenheim

Cover Illustration: iStockphoto/Thinkstock

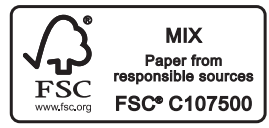




\section{Inhalt}

Vorwort -545

\section{Allgemeine Beiträge}

Nicola Würffel

Hausaufgaben im DaF/DaZ-Unterricht. Ein altes Thema (digital)

neu denken -546

Karin Kleppin

Sprachlernberatung: Hype oder Notwendigkeit? — 571

Daniel Pust

Integrationsmechanismen des Blended Learning im Fremdsprachenunterricht. Eine explorative Fallstudie -586

Christina Winter da Silva

Förderung des kritischen Denkens und des Fremdverstehens im DaF-Unterricht am Beispiel von Texten aus deutschen Straßenzeitungen -602

Benjamin van Well

Lyrik im germanistischen Fremdsprachenunterricht - Entwicklung und Erprobung eines Unterrichtsmodells zum Aufbau von symbolic competence - 618 
